\title{
Research Paper: \\ Comparing the Effect of Exercise Therapy With Kinesio Taping on Pain and Electrical Activity of Muscles in Women With Patellofemoral Pain Syndrome
}

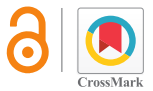

\author{
*Ali Yalfani ${ }^{1}(\mathbb{C})$ Maryam Abdolmaleki $^{1}$ (1), Zahra Raeisi ${ }^{2}$ (1)
}

1. Department of Pathology and Sport Biomechanics, Faculty of Sport Science, Bu-Ali Sina University, Hamedan, Iran.

2. Department of Sport Pathology and Physiology, Faculty of Sport Science, Arak University, Arak, Iran.

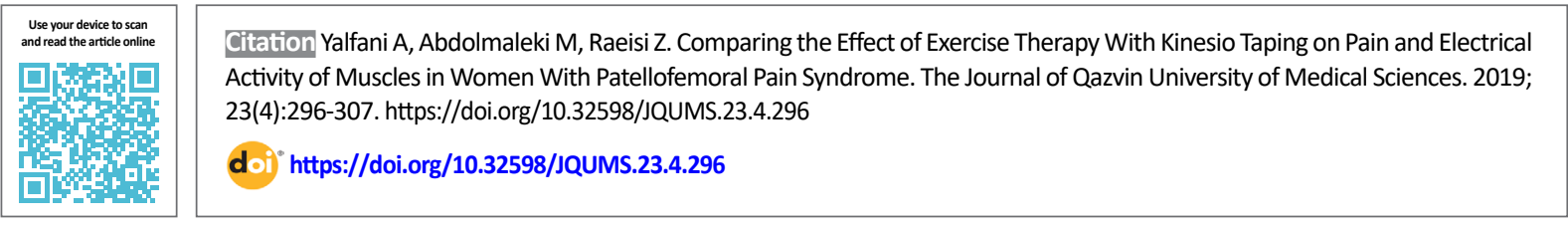

Received: 01 Dec 2018 Accepted: 23 Apr 2019 Available Online: 01 Oct 2019

Keywords: Patellofemoral pain syndrome, Exercise therapy, Athletic tape, Electromyography

\section{A B STR ACT}

Background Patellofemoral pain syndrome (PFPS) is one of the most prevalent knee disorders. In fact, one in four patients with knee disorders suffers from this syndrome.

Objective The purpose of the present study was to compare the efficacy of 8-week exercise therapy with and without Kinesio taping on the pain and vastus medialis oblique/vastus lateralis (VMO/VL) electrical activity ratio in women with PFPS.

Methods In this quasi-experimental study, 45 women with PFPS were participated. They were randomly divided into three groups of exercise therapy, exercise therapy with Kinesio taping, and control. Their pain and muscle activity were evaluated by visual analogue scale and surface electromyography, respectively before and after treatment. Paired t-test and one-way ANOVA were performed for statistical analysis. Findings The results reported a significantly reduced pain and improved VMO/VL muscle electrical activity ratio in both experimental groups $(P<0.05)$ but there was no statistically significant difference between both groups in reducing pain $(P>0.05)$. Moreover, more reduction in onset delay of the electrical activity of VMO relative to the VL was observed in the group that performed exercises with using Kinesio tapes $(\mathrm{P}<0.05)$.

Conclusion Kinesio taping as an effective complement to exercise therapy is recommended for the improvement of patients with PFPS.

\section{Extended Abstract}

\section{Introduction}

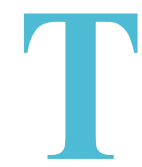

he patellofemoral pain syndrome (PFPS) is a common knee problem, especially in women. Various causes have been found for this multifactorial syndrome including the weakness of quadriceps femoris muscles especially the vastus medialis muscle, abnormal patellar tracking, patella lateralization, hormonal factors, stiffness of lateral structure, shortness of soft tissues around joints, overuse of muscles, and muscle imbalance between vastus medialis oblique (VMO) and vastus lateralis (VL) muscles $[1,4,5]$.

There is a significant and positive association between delay in onset of VMO relative to VL and lateral patel-

\section{* Corresponding Author:}

Ali Yalfani

Address: Department of Pathology and Sport Biomechanics, Faculty of Sport Science, Bu-Ali Sina University, Hamedan, Iran

Tel: +98 (81) 38381422

E-Mail: ali_yalfani@yahoo.com 
lofemoral dislocation in patients with PFPS. Delay in the activity onset of VMO causes abnormal patellar dislocation and increases the stress of patellofemoral joint leading to the patellofemoral pain. Given the attributed advantages of tapes for the onset of muscle activities and the importance of the onset time of quadriceps femoris muscles in patients with PFPS, the use of tapes along with exercise can be a non-invasive and effective technique to accelerate muscle recruitment in patients if the tape can affect the activation onset of target muscles [1]. In this regard, the present study aimed to compare the impact of exercise therapy consisting of a selected protocol with and without kinesio taping (KT) on the pain relief and reduction of delay in the electrical activity onset of VMO relative to VL in women with the PFPS.

\section{Materials and Methods}

In this quasi-experimental study, 45 women with PFPS who referred to the Physical Medicine and Physiotherapy Clinic in Hamedan were voluntarily participated. The inclusion criteria were: Knee pain while climbing up and down the stairs and kneeling and sitting with knees bent for a long time; no history of surgery, hit, neurological, musculoskeletal, and skeletal diseases in the lower extremity; and positive Patellar Apprehension and Clarke's tests [1, 4, 8]. Participants were randomly divided into three groups of exercise therapy $(n=15)$, exercise therapy plus KT $(n=15)$, and control $(\mathrm{n}=15)$. Intervention was performed in exercise groups for eight weeks, three sessions per week, every other day (so that the recovery is provided).

At the beginning of each exercise session, a 3-minute stationary biking was performed to warm up, and then the strengthening and stretching exercises were performed [4, $16,22,25-32]$. In the exercise group with KT, the taping was performed according to KT method for each participant before the start of exercise. after about 20-30 minutes, the participants were allowed to exercise [33]. The control group did not receive any special treatment during this period. Three patients in the control group could not complete the intervention due to some reasons and then excluded from the study.

Patients' pain was measured by the visual analogue scale and the onset time for the VMO and VL muscles' activity was recorded by an electromyographic device before and after the intervention. Electromyographic signals were recorded during the stair climbing task (stair height $=20 \mathrm{~cm}$ ). The stair climbing task included climbing up three stairs from one side and climbing down from the opposite side. Each participant performed the stair climbing task five times. The mean results were recorded and used as a final data.

\section{Results}

The demographic characteristics of participants are shown in Table 1. There was no significant difference between them in terms of demographic characteristics $(\mathrm{P}>0.05)$.

The pain and delay in electrical activity of the VMO relative to VL significantly decreased during climbing up and down stairs after eight weeks of exercise in both experimental groups $(\mathrm{P}<0.05)$, but there was no significant change in the control group after 8 weeks $(\mathrm{P}>0.05)$. Significant differences were found between three study groups $(\mathrm{P}<0.05)$. Results of the Tukey's post hoc test indicated significant lower delayed activity of the VMO relative to VL in the exercise group with $\mathrm{KT}(\mathrm{P}=0.001)$.

\section{Conclusion}

The PFPS decreases the patients' quality of life [3]. The conservative treatment methods for this syndrome includes non-surgical interventions such as resting, using ice, antiinflammatory drugs, hydrotherapy, taping, moderation of activities and exercise therapy $[1,8,9]$.

The abnormal lateral patellofemoral displacement may also decrease due to the improved onset time of muscle activity after eight weeks in two exercise groups; hence,

Table 1. One-way ANOVA results from comparing differences in demographic characteristics between groups

\begin{tabular}{|c|c|c|c|c|c|}
\hline \multirow{2}{*}{ Variables } & \multicolumn{3}{|c|}{ Mean $\pm S D$} & \multirow{2}{*}{$\mathbf{F}$} & \multirow{2}{*}{$\mathbf{P}$} \\
\hline & Exercise With KT ( $n=15)$ & Exercise ( $n=15)$ & Control $(n=12)$ & & \\
\hline Age (year) & $25.53 \pm 3.62$ & $25.33 \pm 5.35$ & $27.42 \pm 4.48$ & 1.176 & 0.319 \\
\hline Height (cm) & $165.6 \pm 4.43$ & $165.47 \pm 3.61$ & $166.83 \pm 4.94$ & 0.375 & 0.690 \\
\hline Weight (kg) & $59.67 \pm 3.48$ & $59.86 \pm 4.83$ & $61.83 \pm 5.30$ & 0.420 & 0.420 \\
\hline
\end{tabular}


pain as a response to abnormal patellofemoral displacement, can decrease simultaneously with the improvement of VMO/VL activity. The pain and delay in the onset of the VMO activity relative to VL was decreased in both groups after exercise, where the reduction was more in the exercise group with KT. This factor can be taken into account as a determinant for the PFPS treatment. Therefore, we suggest multifactorial rehabilitation programs with an emphasis on exercise along with using the supportive equipment such as tapes to improve the conditions of patients with PFPS.

\section{Ethical Considerations}

\section{Compliance with ethical guidelines}

This study was approved by the Research Ethics Committee of Hamedan University of Medical Sciences (Code: IR.UMSHA.REC.1396.842) and registered by Iranian Registry of Clinical Trials (Code: IRCT20181002041203N1). The research goal and method were explained to the participants and they were assured of the confidentiality of their information. They were free to quit the study at any time.

\section{Funding}

This research did not receive any specific grant from funding agencies in the public, commercial, or not-for profit sectors.

\section{Authors' contributions}

Conceptualization, validation, visualization, supervision, project administration, and funding acquisition: Ali Yalfani; Methodology: Ali Yalfani and Maryam Abdolmaleki; Formal analysis: All authors; Investigation, resources, and draft preparation: Maryam Abdolmaleki and Zahra Raeisi; Editing: Ali Yalfani and Zahra Raeisi.

\section{Conflicts of interest}

The authors declared no conflicts of interest. 


\title{
مقايسه تأثير تمريندرمانى باو بدون كينزيوتيب بر ميزان درد و فعاليت الكتريكى عضلهها در زنان مبتلا به سندرم درد كثككى - مائى تمرئى
}

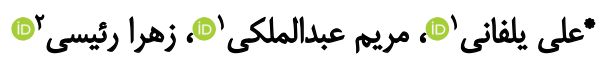 \\ ا. ا. تروه آسيبشناسى و بيومكانيك ورزشى، داتشكده علوم ورزشى، دانشكاه بوعلى سينا، همدان، ايران.

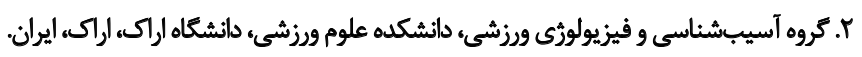

\begin{abstract}
حيكيد
إتمينه سئدرم درد كشككى - رانى يكى از شايعترين اختلالات مفصل زانوست كه از هر جهار نفر مبتلابه درد زانو، يك نفر از اين عارضه.

رنج ميرد.

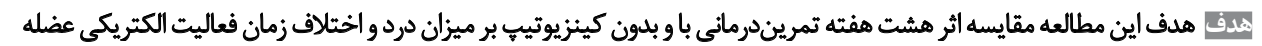

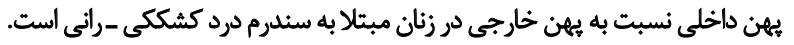

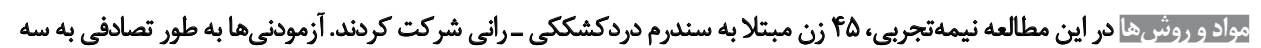

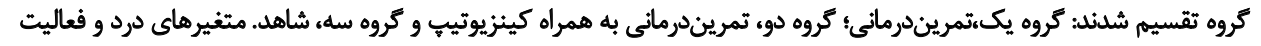

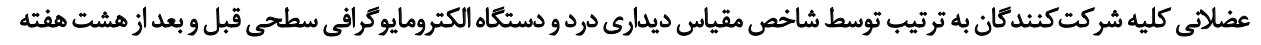

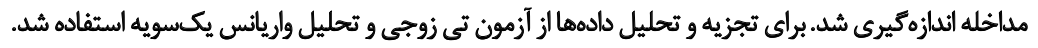

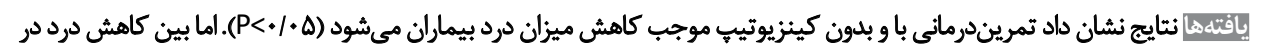

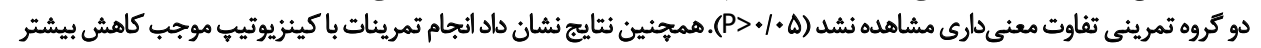

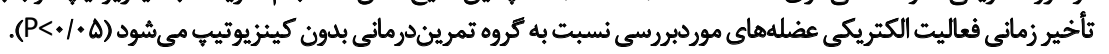

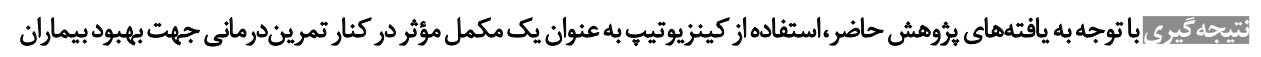

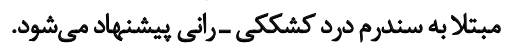

تاريخ دريافت: • •1 آذر

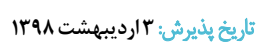

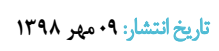

كليدوأوهائ

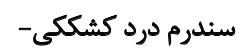

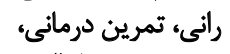
تيب ورزشي، فعاليت تئي الكتريكى عضله ها تالت

خط اول در درمان سندرم درد كشككى - رانى مديريت

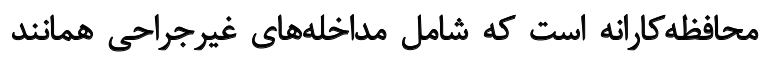
مقدمه

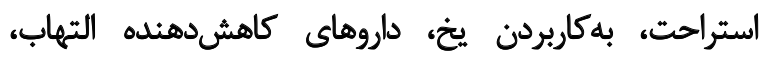

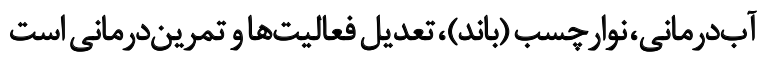

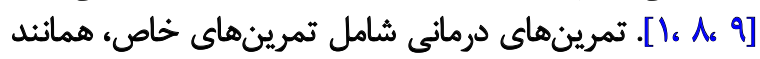

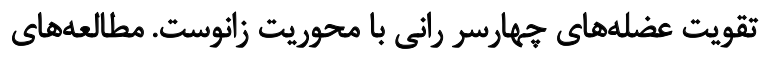

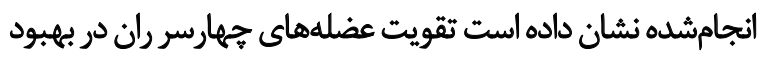

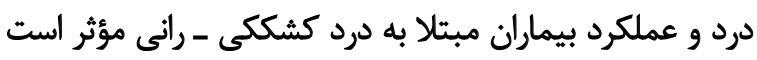

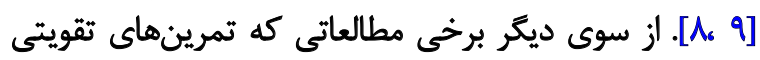

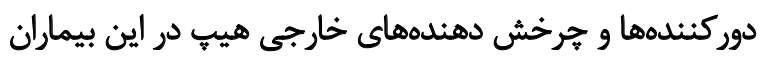

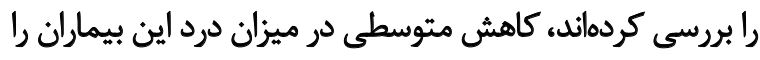

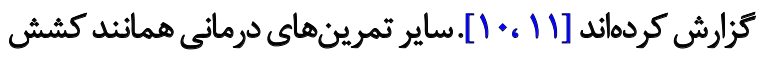

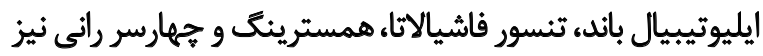

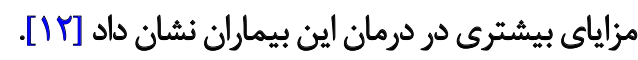

سندرم درد كشككى -رانى با احساس درد در كشكك يا بيرامون آن،

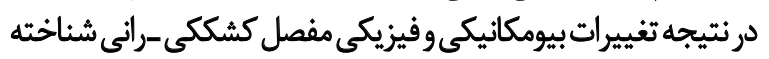

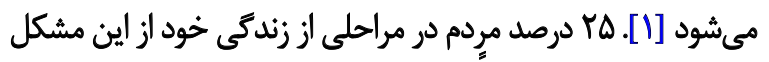

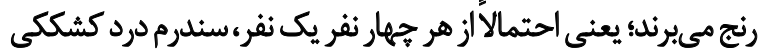

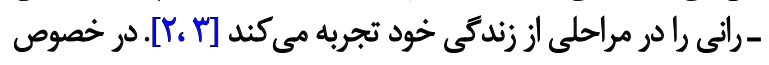

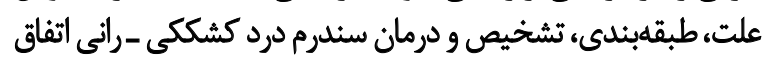

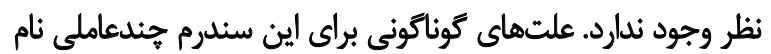

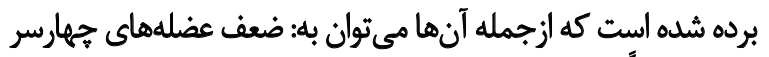

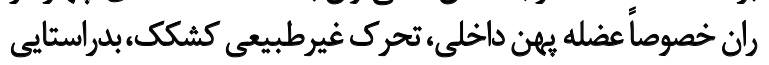

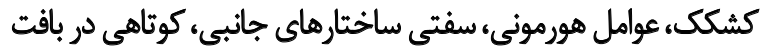

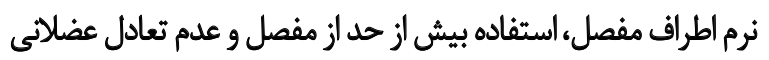

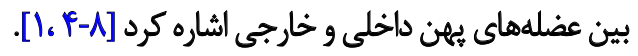
-

* عويسينده مسيئول: على يلفانى

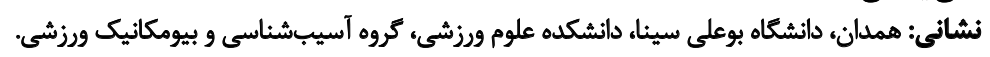
تلفن: رايانامه: ali_yalfani@yahoo.com 
به فعاليت عضلههاى هدف، استفاده از نوارجسب در كنار تمرينات

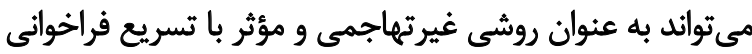

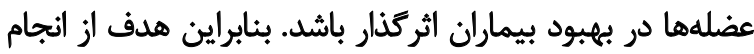

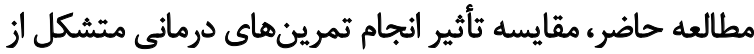

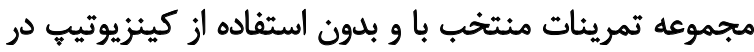

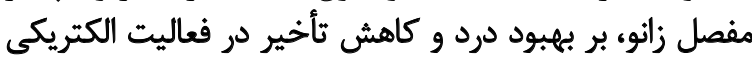

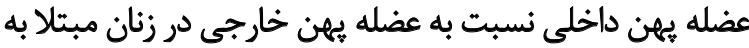
سندرم درد كشككى -رانى است.

\section{مواد وروشها}

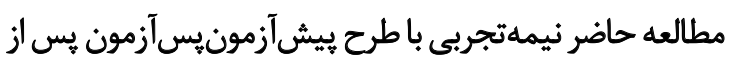

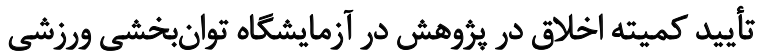

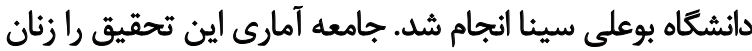

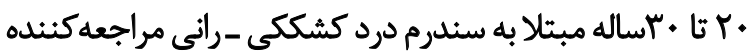
به درمانكاه طب فيزيكى و فيزيوترايى تشكيل داديند. معيارهاى ورود افراد: وجود درد زانو در حين بالاو واييين رفتن

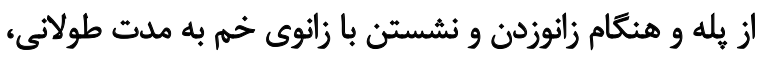

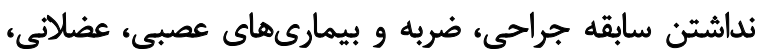

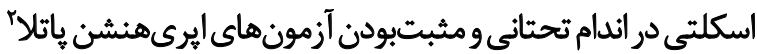

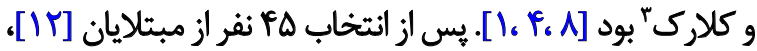

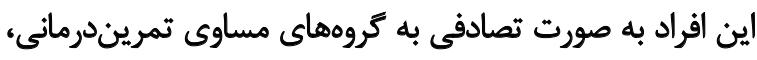

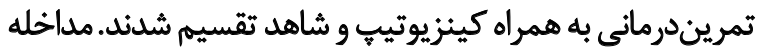

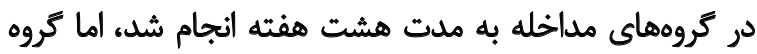

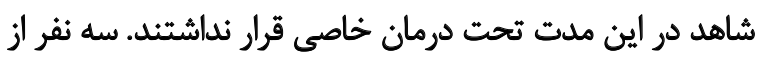

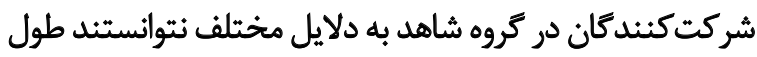
دوره هشت هفته را به اتمام برسانند و از مطالعه خارج شارئ شنداند. سيخنال هاى الكترومايوگرافى در اين تحقيق در حين تكليف

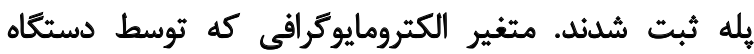
الكترومايوكرافى سطحى (ساخت شركت مكاوين كشور فنلاند)

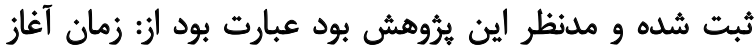

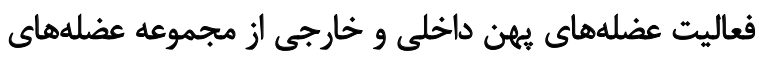

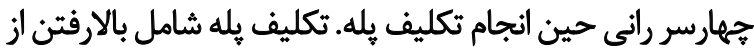

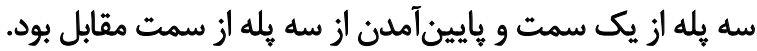

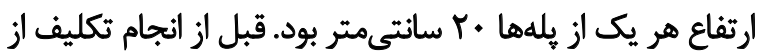

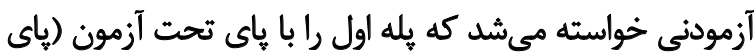

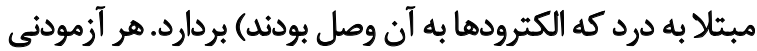

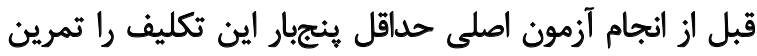

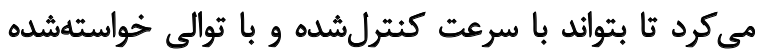

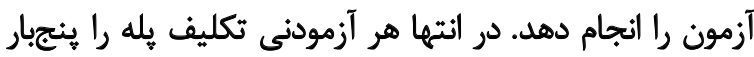

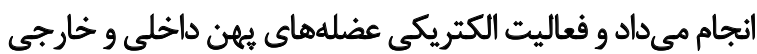
از مجموعه عضلههاى جهارسر رانى در حين اين بنج تكرار ثبت داختي

2. Patellar Apprehension

3. Clark
عضله بيهن داخلى تنها ثباتدهنده فعال داخلى مفصل

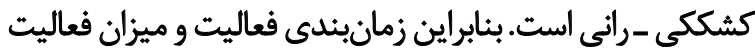

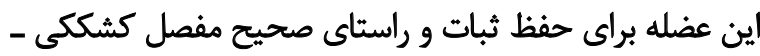

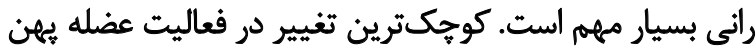

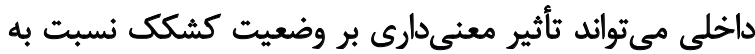

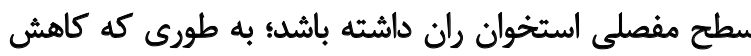

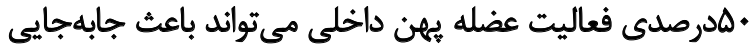

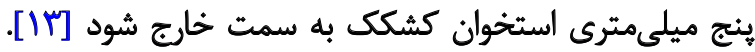

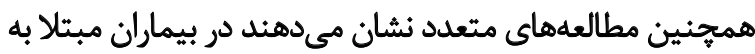

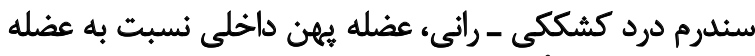

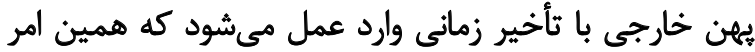

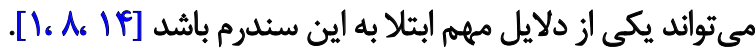

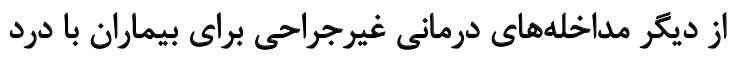

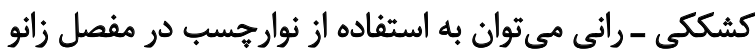

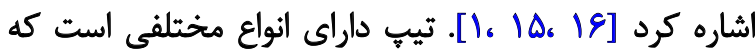

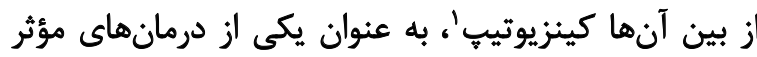

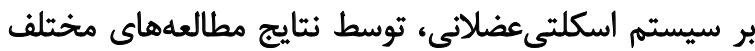

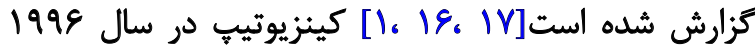

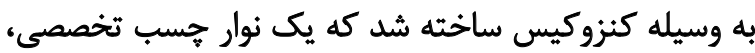

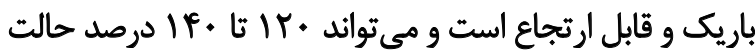

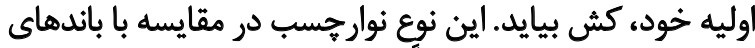

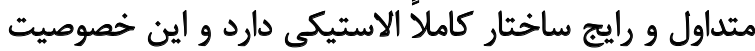

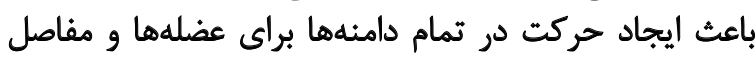

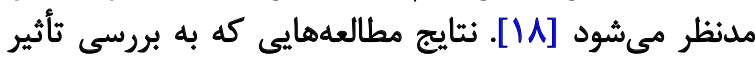

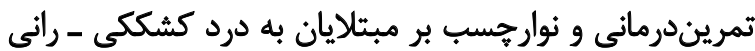

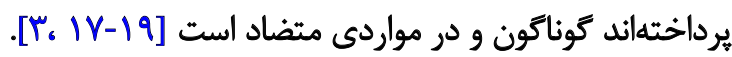
عليرغم درمانهاى انجامشده همجنان بيماران از درد و كاهش

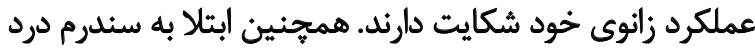

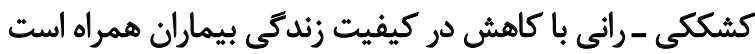

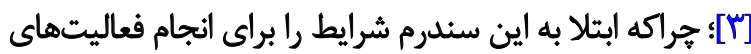

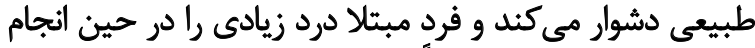

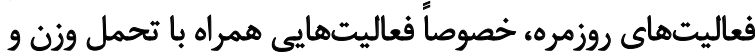

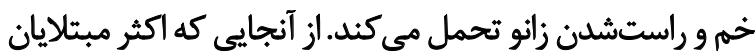

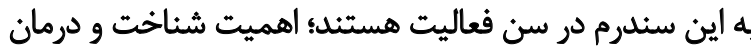

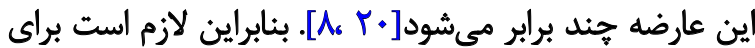

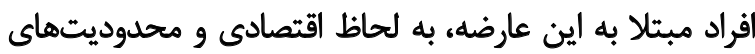

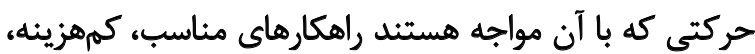
بدون عوارض و در عين حال قابلاجرا ييشنهاد شود.

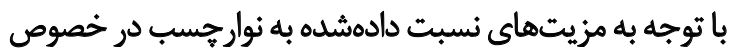

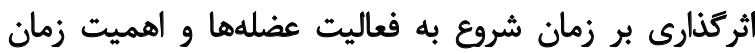

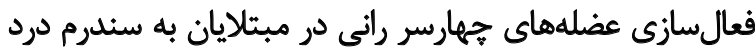

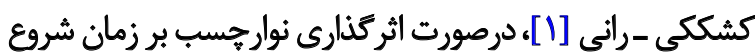

1. Kinesiotape 
ايزومتريك هجهارسر راني"، اكستنشن انتهايي زانو"، بالآوردن

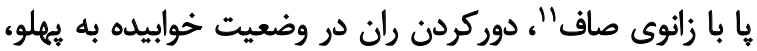

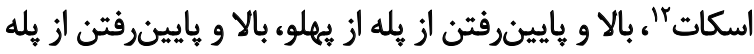

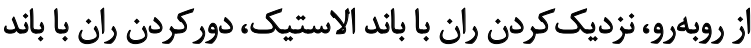

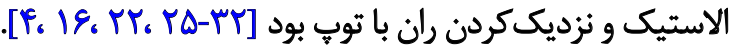

در گروه مداخله به همراه كينزيوتيب قبل از شروع تمرينات

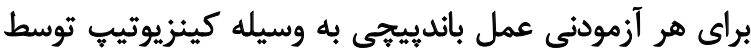

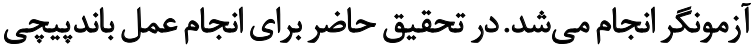

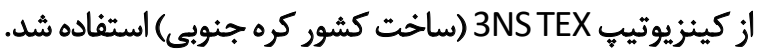

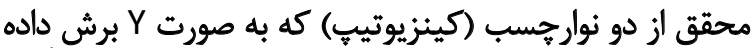

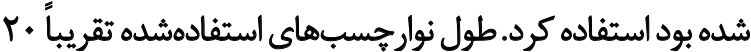

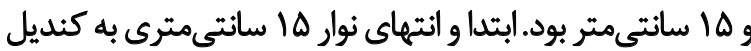

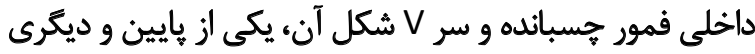

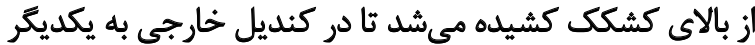

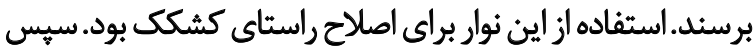

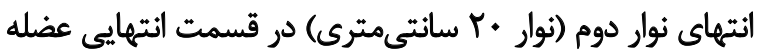

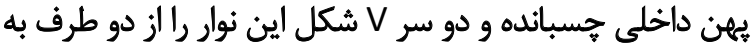

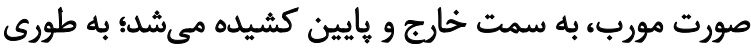

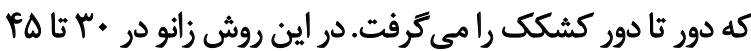

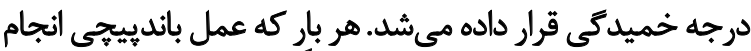

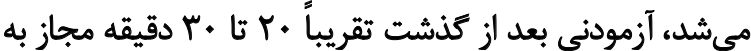

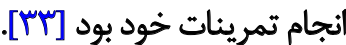

يس از تمرينات كششى، برنامه تمرينى كه براي هر دو كروه

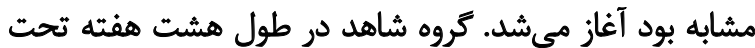

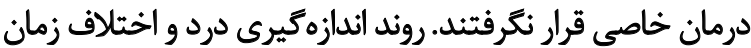

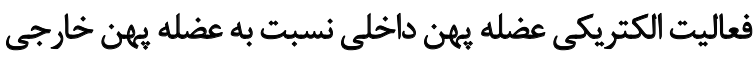
بس از گذشت هشت هفته در هر سه گروه تكرار شد.

جهت انجام تجزيه و تحليل آمارى در آغاز براى بررسى توزيع

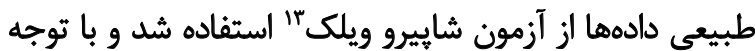

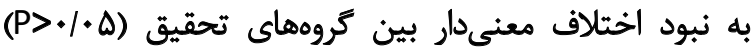

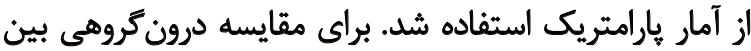

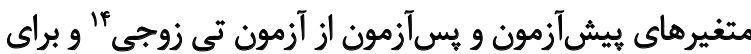

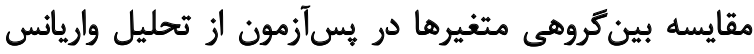

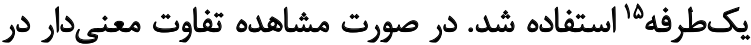

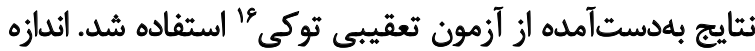

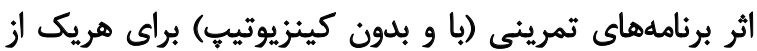

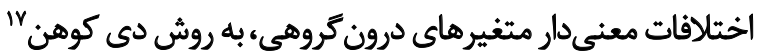

9. Isometric contraction quadriceps

10. Terminal knee extension

11. Straight leg raising

12. Squat

13. Shapiro-wilk

14. Paired samples t-test

15. One-way ANOVA

16. Tukey post hoc test

17. Cohen's d

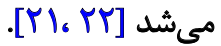

در اين يُوهش جهت محاسبه زمان آغاز فعاليت عضلههاى

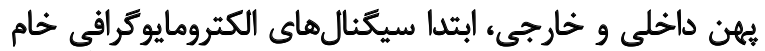

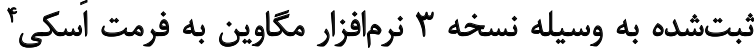

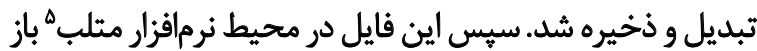

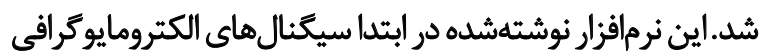

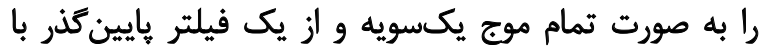

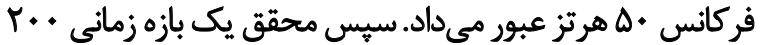

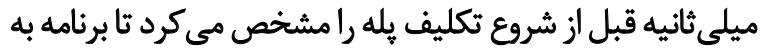

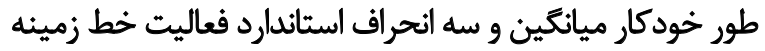

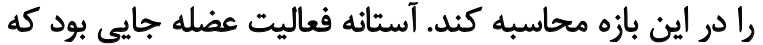

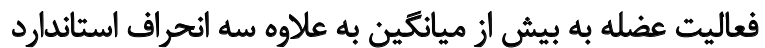

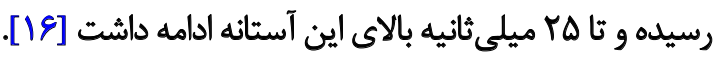

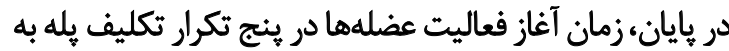

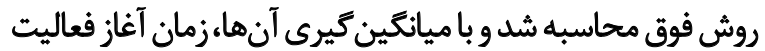

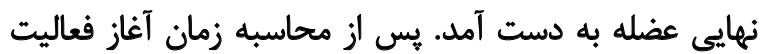

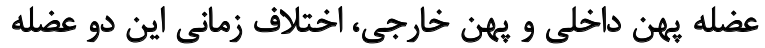

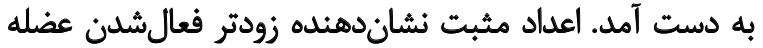

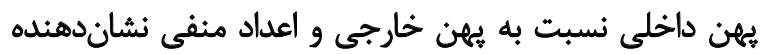

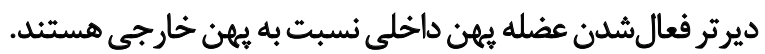

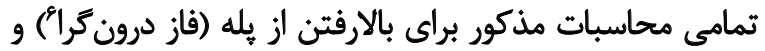

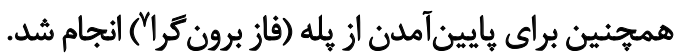

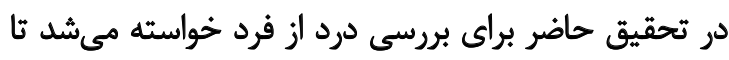

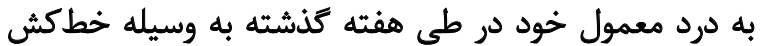

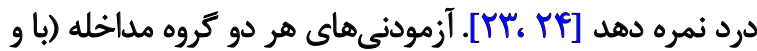

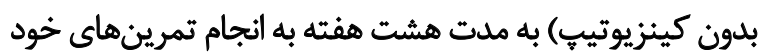

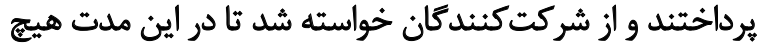

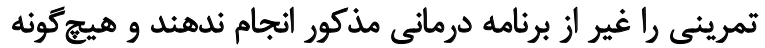

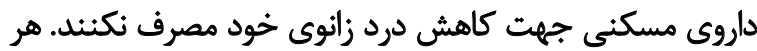

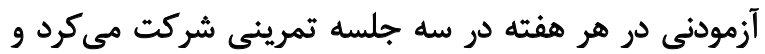

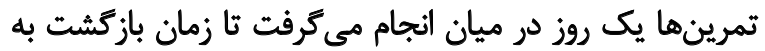

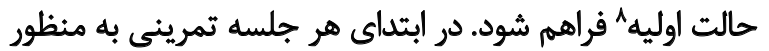

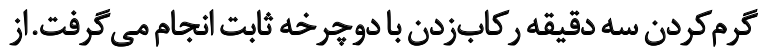

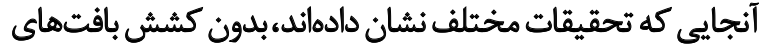

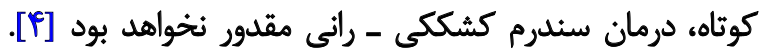

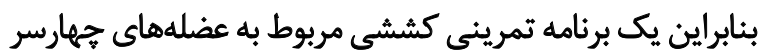

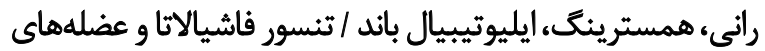

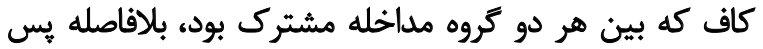

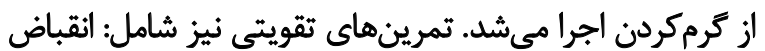
4. ASCII
5. Matlab software
6. Concentric
7. Eccentric
8. Recovery 


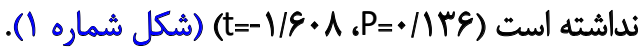
همجنين نتايج آزمون تى زوجى نشان داد يس از هشت هفته

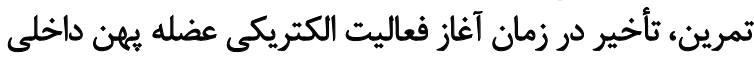

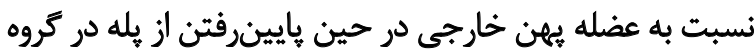

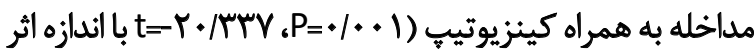

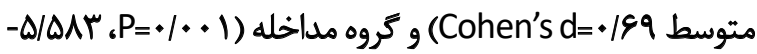

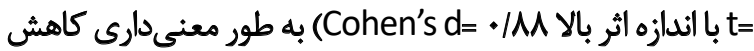

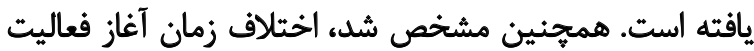

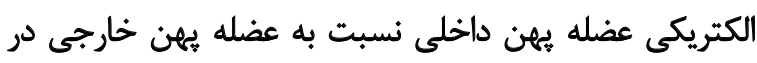

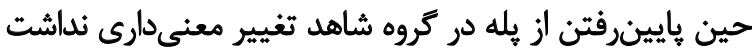

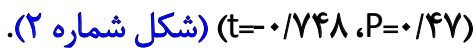

نتايج تحليل واريانس يكطرفه در يس آزمون نشان داد در ميزان

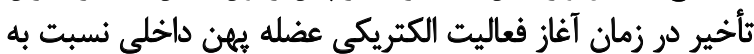

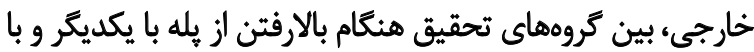

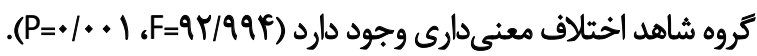

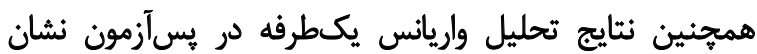

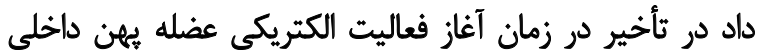

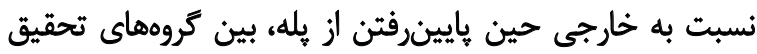

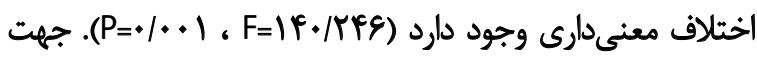

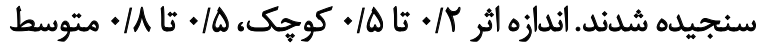

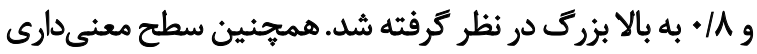

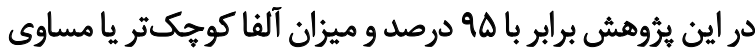

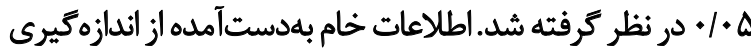

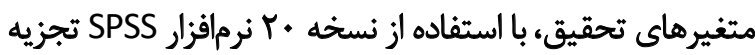

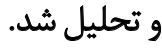

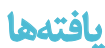

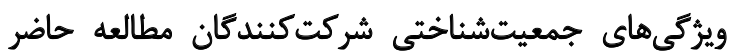

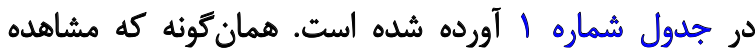

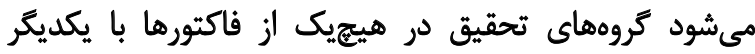

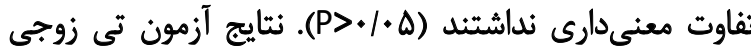

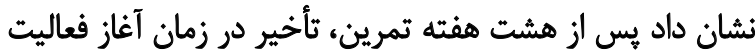

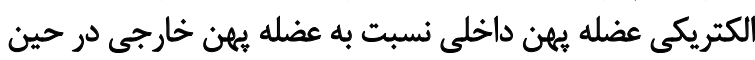

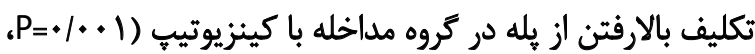

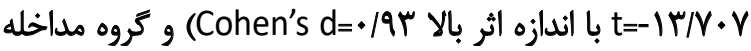

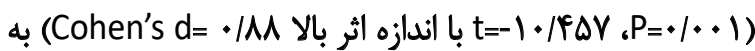

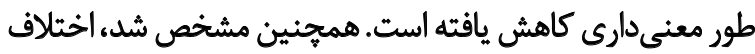

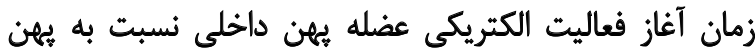

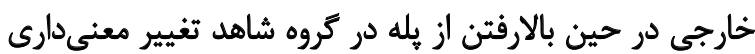

جدول ا. نتايج مربوط به آزمون تحليل واريانس يكطرفه جهت بررسى وجود اختلاف در ويزكى هاى جمعيتشناختى بين كروهها

\begin{tabular}{|c|c|c|c|c|c|}
\hline \multicolumn{5}{|c|}{ ميانكينداتحراف معيار } & \multirow[b]{2}{*}{ متغير } \\
\hline سطح معنى دارى & $\mathbf{F}$ & شاهد (n=1r) & مداخله (10) & تمرين و كينزيوتيب (n=10) & \\
\hline.$/ 419$ & $1 / 1 v 8$ & $T V / T T \pm F / P A$ & $r \Delta / r T \pm \Delta / T \Delta$ & $r \Delta / \Delta T \pm T / Q T$ & سن (سال) \\
\hline Ira. &.$/$ rva & IEg/Arte/qf & $|\varnothing Q / F V \pm r / 9|$ & $180 / 9 . \pm t / 4 T$ & قد (سانتىمتر) \\
\hline.$/ F T$. & $+/ T$ & $g / / \Lambda \mu \pm \Delta / \mu^{*}$ & $\Delta V A E T F / A T$ & $\Delta Q / 8 V \pm T / F \Lambda$ & وزن (كيلوكرم) \\
\hline
\end{tabular}

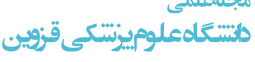

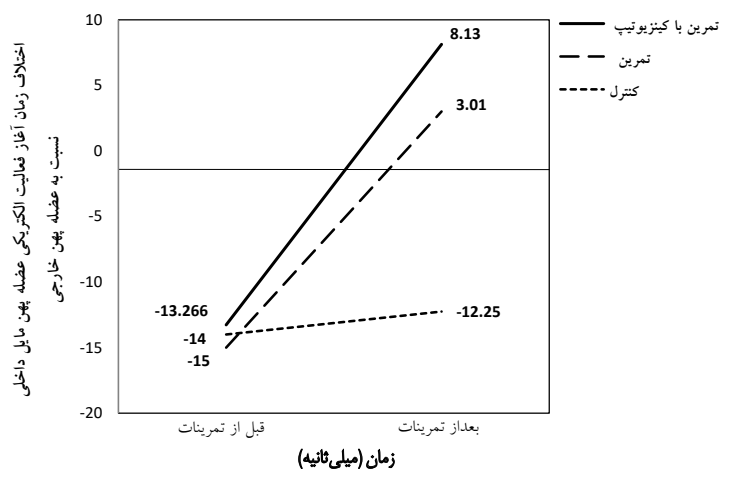

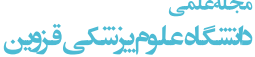

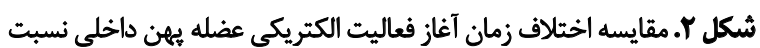

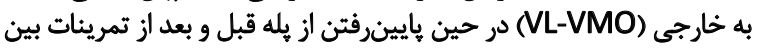

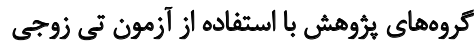

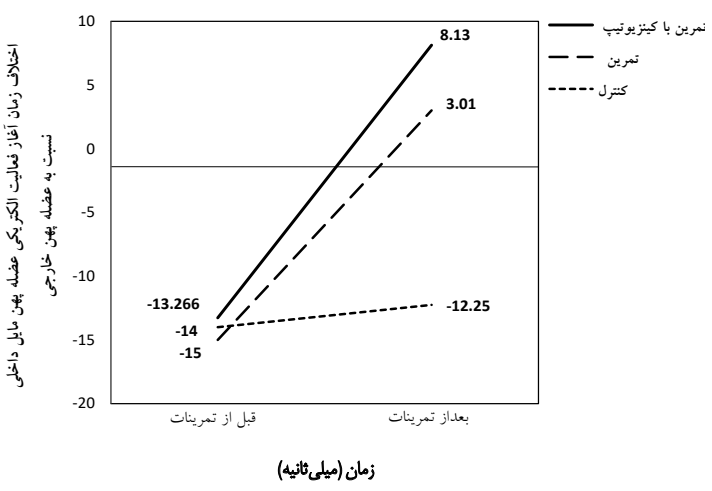

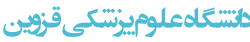

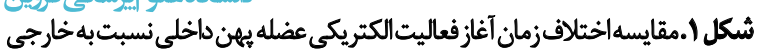

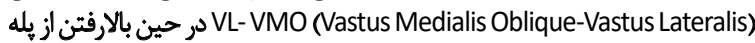
قبل و بعد أز تمريئات بين كروهماى يرؤوهش با استفاده أز آزمون تى زوجى 
جدول זّ. مقايسه اختلاف زمان آغاز فعاليت الكتريكى عضله يُهن داخلى نسبت به خارجى (VL- VMO) در حين انجام تكليف يُله در سه كروه با استفاده از آزمون

مداخله و كروه شاهد اختلاف وجود دارد و كاهش تأخير زمانى

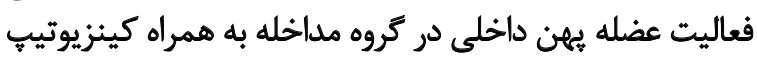

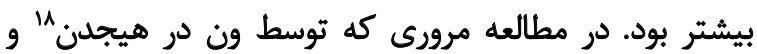

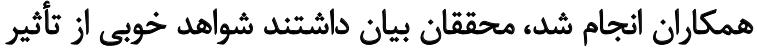

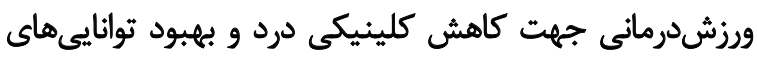

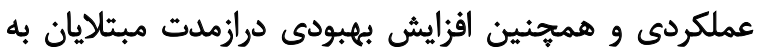

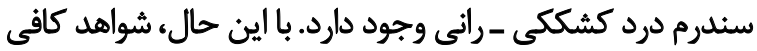

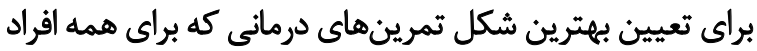

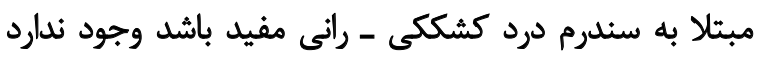

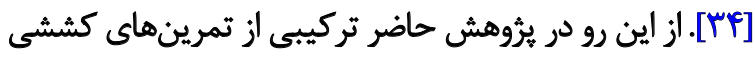

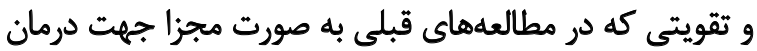

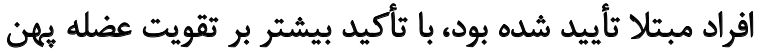

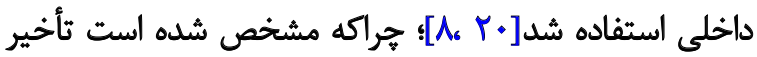

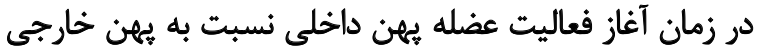

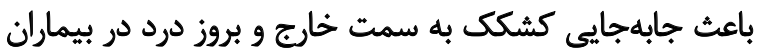

مىشود [1T]

نتايج بهدستآمده اثربخشى اين مجموعه تمرينى بر كاهش

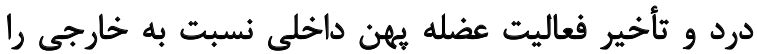

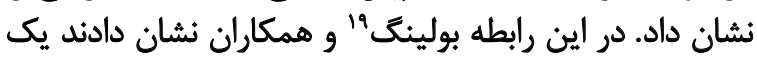

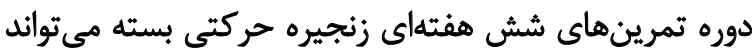

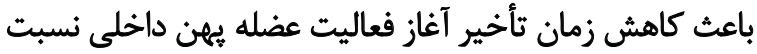

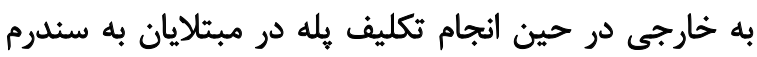

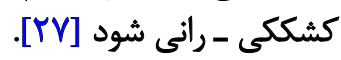

برهمرخوردن تعادل بين زمان آغاز فعاليت عضله بهين داخلى سلى

18. Van der Heijden

19. Boling
مشخص كردن اختلاف بين كروهها از آزمون تعقيبى توكى استفاده و نتايج در جدول شماره ب آورده شده است كرون.

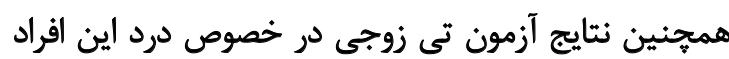

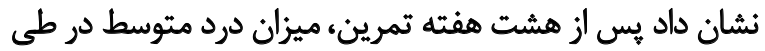

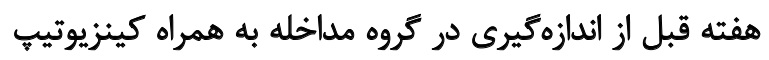

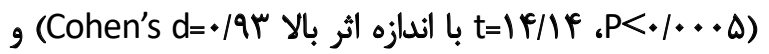

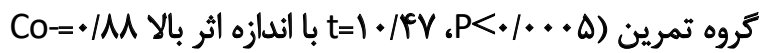
بهen's d

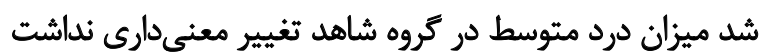

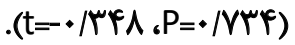

نتايج تحليل واريانس يكطرفه ميزان درد در يسآزمون

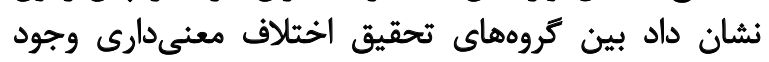
دارد (F=VA/FY

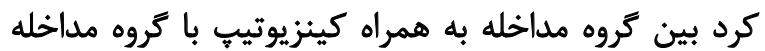

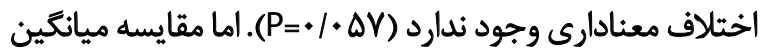

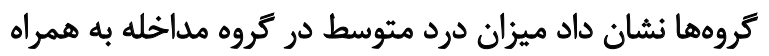

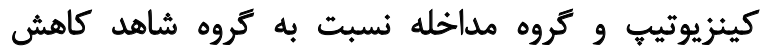

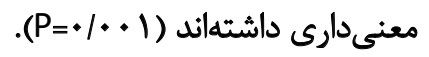

\section{بحث و نتيجنه كيرى}

نتايج مطالعه حاضر نشان داد بـ إز هشت هفته تمرين در هر دو تروه مداخله، درد و تأخير زمان آغاز فعاليت الكتريكى عضل عضله

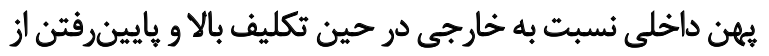

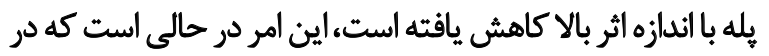

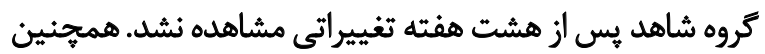
مشخص شد بين كروه مداخله به همراه كينزيوتيثي با كروه 


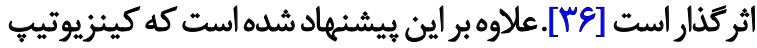

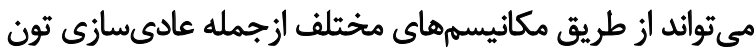

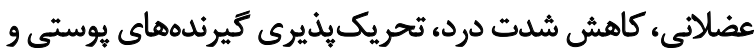

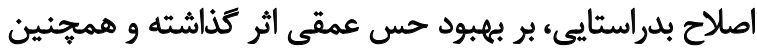

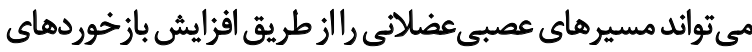

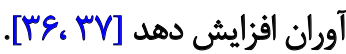

لوكان و همكاران در نتايج مطالعه مرورى خود بيان كردند

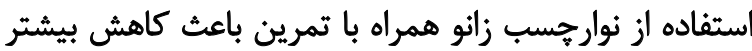

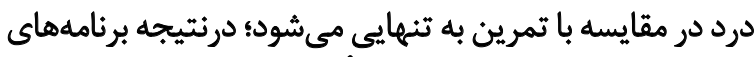

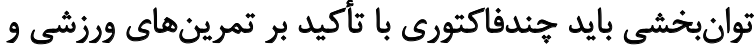

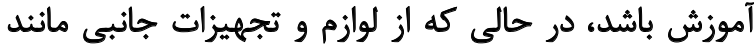

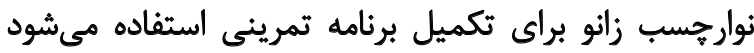

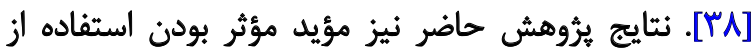

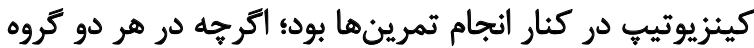

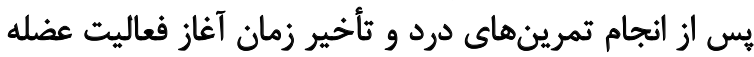

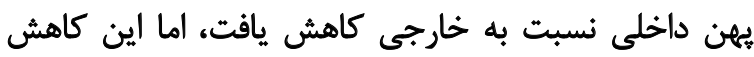

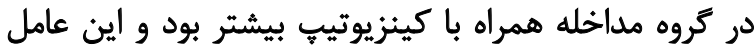

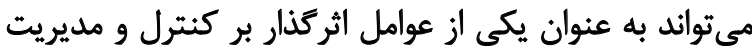
سندرم درد كشككى ـ رانى به حساب آيد عائ.

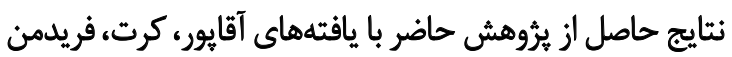

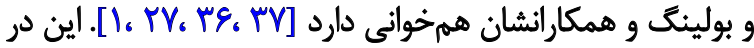

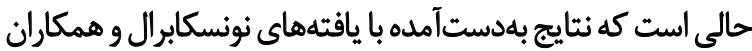

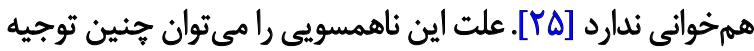

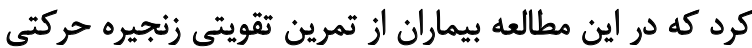

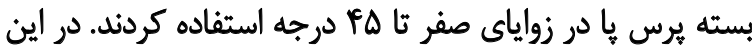

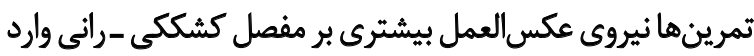

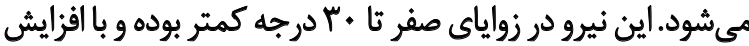

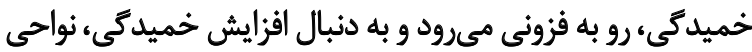

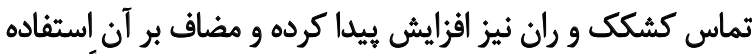

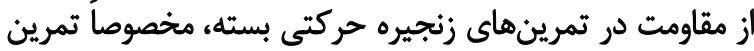

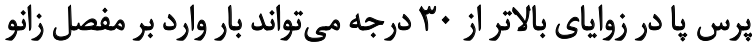

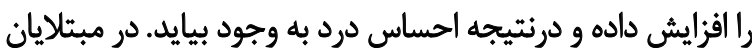

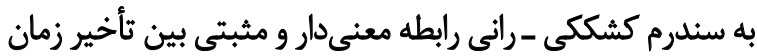

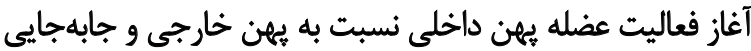

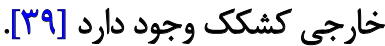

آغاز فعاليت تأخيرى عضله بهابهن داخلى نسبت به به خارجي

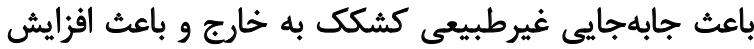

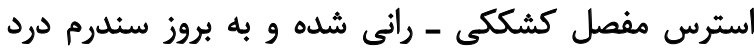

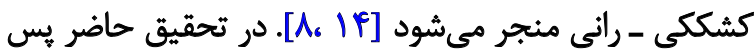

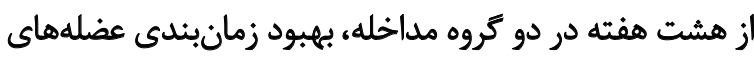

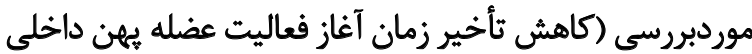
نسبت به خارجى) مشاهده شد (كاهير زمان أغاز احتمال دارد جابهجايى خارجى غيرطبيعى كشكى در
نسبت به خارجى نشان از اختلال در كنترل حركتى و عصبى در

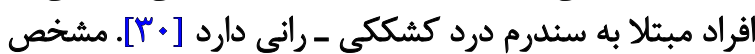

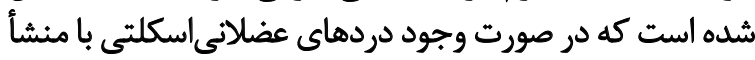

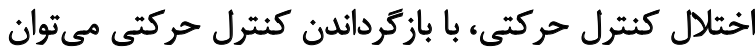

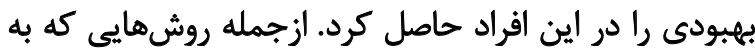

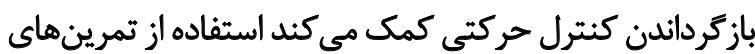

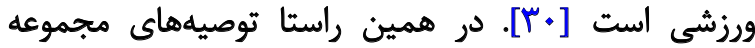

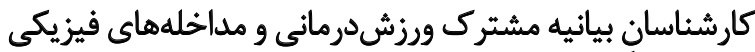

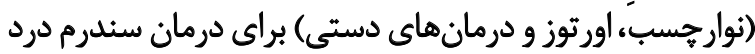

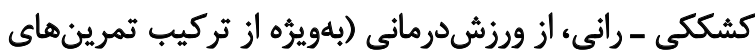

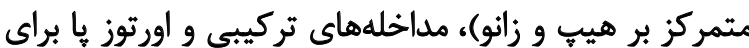

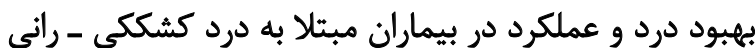

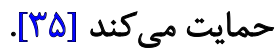
نتايج حاضر نيز نشان داد با استفاده از تمرينهاى ورزشى و

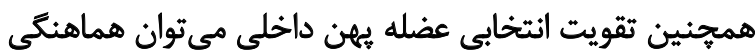

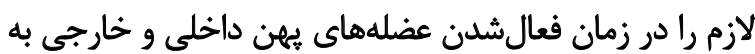

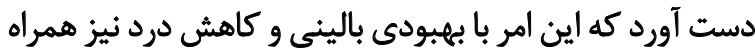

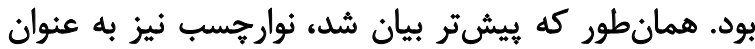

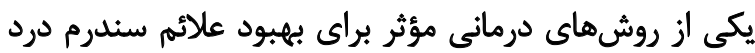

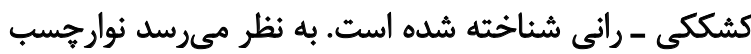

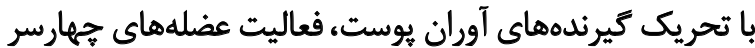

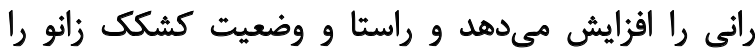

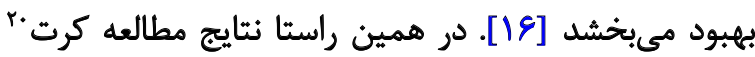

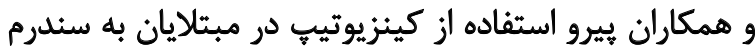

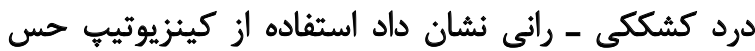

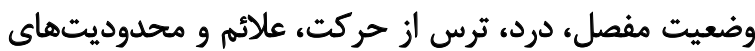

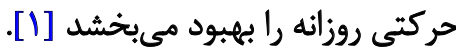

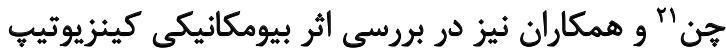

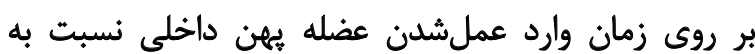

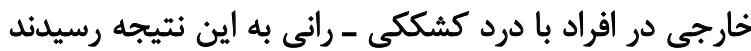

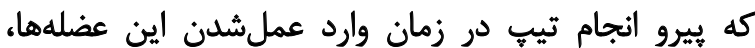

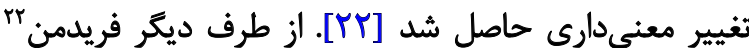

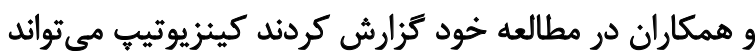

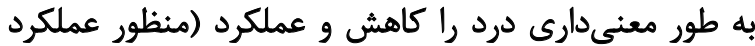

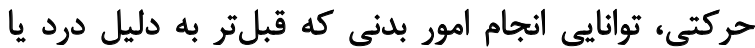

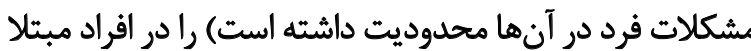

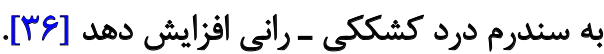
استفاده از كينزيوتيب مي تواند تحريكيذيرى عضله را افزايش

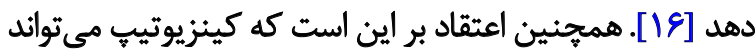
كردش خون را افزايش دهد كه به نوبه خود بر عملكرد عضلهها

20. Kurt

21. Chen

22. Freedman 


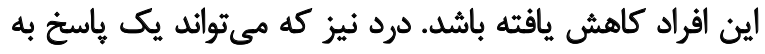

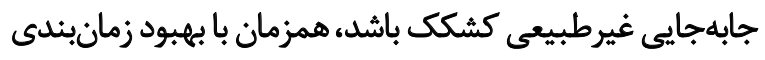

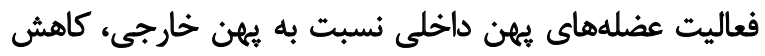

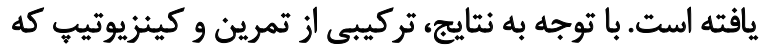

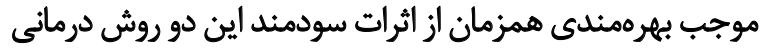

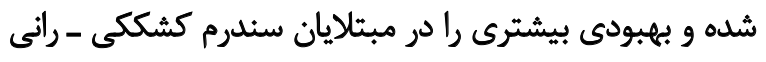

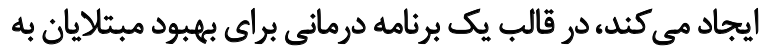

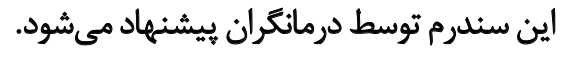

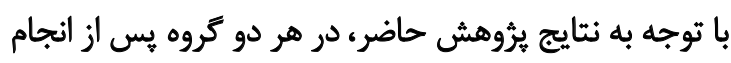

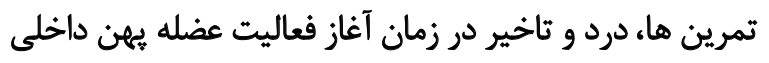

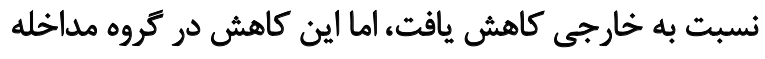

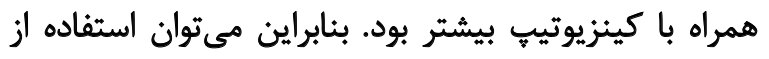

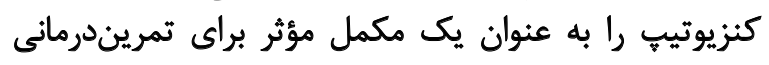

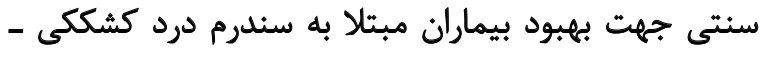
رانى بيشنهاد داد.

مالامظاته أخلاقي

\section{بيروى از اصول اخلاق بروهش}

اين مطالعه با كد اخلاق

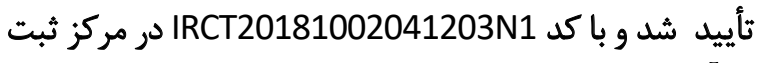

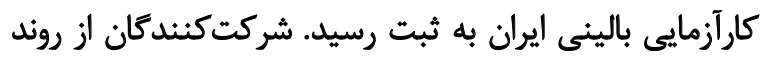

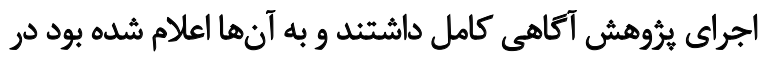

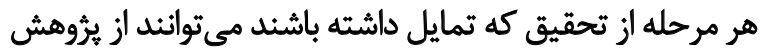
خارج شوند.

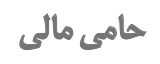

مطالعه حاضر بركرفته از هايان نامه كارشناسىارشد خانم مريم

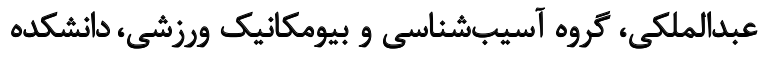

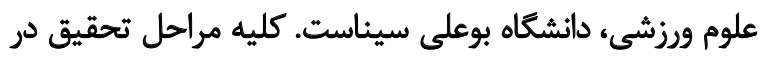
آزمايشعَاه توانبخشى ورزشى دانشعاه بوعلى بوسيناى همدان انجام شده است.

$$
\text { مشاركت ثويسندكّان }
$$

مفهومسازى: على يلفانى، روششناسى: على على يلفانى، مريم

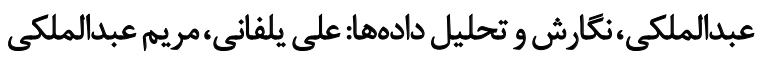

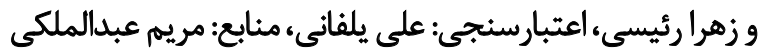

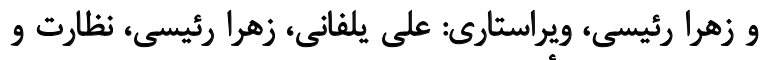
مديريت يرورٌه و تأمين مالى: على يلفانى.

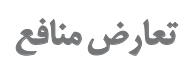

نويسندكان اعلام مى كنند مقاله حاضر تعارض منافع ندارد. 


\section{References}

[1] Kurt EE, Büyükturan Ö, Erdem HR, Tuncay F, Sezgin H. Shortterm effects of kinesio tape on joint position sense, isokinetic measurements, and clinical parameters in patellofemoral pain syndrome. J Phys Ther Sci. 2016; 28(7):2034-40. [DOI:10.1589/ jpts.28.2034] [PMID] [PMCID]

[2] Cichanowski HR, Schmitt JS, Johnson RJ, Niemuth PE. Hip strength in collegiate female athletes with patellofemoral pain. Med Sci Sports Exerc. 2007; 39(8):1227-32. [DOI:10.1249/ mss.0b013e3180601109] [PMID]

[3] LaBella C. Patellofemoral pain syndrome: Evaluation and treatment. Prim Care Clin Off Pract. 2004; 31(4):977-1003. [DOI:10.1016/j.pop.2004.07.006] [PMID]

[4] Piva SR, Fitzgerald K, Irrgang JJ, Jones S, Hando BR, Browder $D A$, et al. Reliability of measures of impairments associated with patellofemoral pain syndrome. BMC Musculoskelet Disord. 2006; 7:33. [DOI:10.1186/1471-2474-7-33] [PMID] [PMCID]

[5] Yildiz Y, Aydin T, Sekir U, Cetin C, Ors F, Alp Kalyon T. Relation between isokinetic muscle strength and functional capacity in recreational athletes with Chondromalacia patellae. $\mathrm{Br} J$ Sports Med. 2003; 37(6):475-9. [DOI:10.1136/bjsm.37.6.475] [PMID] [PMCID]

[6] Cowan SM, Crossley KM. Does gender influence neuromotor control of the knee and hip? J Electromyogr Kinesiol. 2009; 19(2):276-82. [DOI:10.1016/j.jelekin.2007.07.009] [PMID]

[7] Grelsamer RP. Patellar malalignment. J Bone Joint Surg Am. 2000; 82(11):1639-50. [DOI:10.2106/00004623-20001100000019]

[8] Yalfani A, Raisi Z. Comparison of two methods quadriceps muscle strengthening on land and in the water on pain, function, static and dynamic balance in females with Patellofemoral Pain Syndrome. Sport Sci Res Inst. 2018; 5(13):91-108. [In Persian]

[9] Chevidikunnan MF, Al Saif A, Gaowgzeh RA, Mamdouh KA. Effectiveness of core muscle strengthening for improving pain and dynamic balance among female patients with patellofemoral pain syndrome. J Phys Ther Sci. 2016; 28(5):1518-23. [DOI:10.1589/jpts.28.1518] [PMID] [PMCID]

[10] Santos TRT, Oliveira BA, Ocarino JM, Holt KG, Fonseca ST. Effectiveness of hip muscle strengthening in patellofemoral pain syndrome patients: A systematic review. Brazilian J Phys Ther. 2015; 19(3):167-76. [DOI:10.1590/bjpt-rbf.2014.0089] [PMID] [PMCID]

[11] Alba-Martín P, Gallego-Izquierdo T, Plaza-Manzano G, Romero-Franco N, Núñez-Nagy S, Pecos-Martín D. Effectiveness of therapeutic physical exercise in the treatment of Patellofemoral pain syndrome: A systematic review. J Phys Ther Sci. 2015; 27(7):2387-90. [DOI:10.1589/jpts.27.2387] [PMID] [PMCID]

[12] Peng HT, Song CY. Predictors of treatment response to strengthening and stretching exercises for patellofemoral pain: An examination of patellar alignment. Knee. 2015; 22(6):4948. [DOI:10.1016/j.knee.2014.10.012] [PMID]
[13] Kaya D, Callaghan MJ, Ozkan H, Ozdag F, Atay OA, Yuksel I, et al. The effect of an exercise program in conjunction with short-period patellar taping on pain, electromyogram activity, and muscle strength in patellofemoral pain syndrome. Sports Health. 2010; 2(5):410-6. [DOI:10.1177/1941738110379214] [PMID] [PMCID]

[14] Wong Y. Recording the vastii muscle onset timing as a diagnostic parameter for Patellofemoral pain syndrome: Fact or fad? Phys Ther Sport. 2009; 10(2):71-4. [DOI:10.1016/j. ptsp.2009.02.001] [PMID]

[15] Kettunen JA, Harilainen A, Sandelin J, Schlenzka D, Hietaniemi K, Seitsalo S, et al. Knee arthroscopy and exercise versus exercise only for chronic patellofemoral pain syndrome: A randomized controlled trial. BMC Med. 2007; 5(38):1-8. [DOI:10.1186/1741-7015-5-38] [PMID] [PMCID]

[16] Fagan V, Delahunt E. Patellofemoral pain syndrome: A review on the associated neuromuscular deficits and current treatment options. Br J Sports Med. 2008; 42(10):489-95. [DOI:10.1136/bjsm.2008.046623] [PMID]

[17] Crossley K, Bennell K, Green S, Cowan S, McConnell J. Physical Therapy for Patellofemoral Pain. Am J Sports Med. 2002; 30(6):857-65. [DOI:10.1177/03635465020300061701] [PMID]

[18] Salsich GB, Brechter JH, Farwell D, Powers CM. The effects of patellar taping on knee kinetics, kinematics, and vastus lateralis muscle activity during stair ambulation in individuals with patellofemoral pain. J Orthop Sport Phys Ther. 2002; 32(1):310. [DOI:10.2519/jospt.2002.32.1.3] [PMID]

[19] McEwan I, Herrington L, Thom J. The validity of clinical measures of patella position. Man Ther. 2007; 12(3):226-30. [DOI:10.1016/j.math.2006.06.013] [PMID]

[20] Clijsen R, Fuchs J, Taeymans J. Effectiveness of exercise therapy in treatment of patients with patellofemoral pain syndrome: Systematic review and meta-analysis. Phys Ther. 2014; 94(12):1697-708. [DOI:10.2522/ptj.20130310] [PMID]

[21] Ng GY, Cheng JM. The effects of patellar taping on pain and neuromuscular performance in subjects with patellofemoral pain syndrome. Clin Rehabil. 2002; 16(8):821-7. [DOI:10.1191/0269215502cr563oa] [PMID]

[22] Chen PL, Hong WH, Lin CH, Chen WC. Biomechanics effects of kinesio taping for persons with patellofemoral pain syndrome during stair climbing. Heidelberg: Springer; 2008.

[23] Ganji B. The effect of strengthening of hip abductor and external rotator muscles on pain and function in women with patellofemoral pain syndrome. J Res Sport Rehabil. 2015; 2(4):1-10. [In Persian]

[24] Green ST. Patellofemoral syndrome. J Body W Mov Ther. 2005; 9(1):16-26. [DOI:10.1016/j.jbmt.2003.12.001]

[25] Nunes Cabral CM, Maria De Oliveira Melim Â, De Camargo I, Sacco N, Marques AP. Effect of a closed kinetic chain exercise protocol on patellofemoral syndrome rehabilitation. Paper presented at: $25^{\text {th }}$ International Symposium on Biomechanics in Sports. 23-27 August 2007; Ouro Preto, Brazil.

[26] Fukuda TY, Rossetto FM, Magalhães E, Bryk FF, Garcia Lucareli PR, De Almeida Carvalho NA. Short-term effects of hip 
abductors and lateral rotators strengthening in females with Patellofemoral Pain Syndrome: A randomized controlled clinical trial. J Orthop Sport Phys Ther. 2010; 40(11):736-42. [DOI:10.2519/jospt.2010.3246] [PMID]

[27] Boling MC, Bolgla LA, Mattacola CG, Uhl TL, Hosey RG. Outcomes of a weight-bearing rehabilitation program for patients diagnosed with patellofemoral pain syndrome. Arch Phys Med Rehabil. 2006; 87(11):1428-35. [DOI:10.1016/j. apmr.2006.07.264] [PMID]

[28] Irish SE, Millward AJ, Wride J, Haas BM, Shum GL. The effect of closed-kinetic chain exercises and open-kinetic chain exercise on the muscle activity of vastus medialis oblique and vastus lateralis. J Strength Cond Res. 2010; 24(5):1256-62. [DOI:10.1519/JSC.0b013e3181cf749f] [PMID]

[29] Piva S, Fitzgerald G, Wisniewski S, Delitto A. Predictors of pain and function outcome after rehabilitation in patients with patellofemoral pain syndrome. J Rehabil Med. 2009; 41(8):60412. [DOI:10.2340/16501977-0372] [PMID]

[30] Crossley KM, Cowan SM, Bennell KL, McConnell J. Knee flexion during stair ambulation is altered in individuals with patellofemoral pain. J Orthop Res. 2004; 22(2):267-74. [DOI:10.1016/j.orthres.2003.08.014] [PMID]

[31] Hodges PW. Pain and motor control: From the laboratory to rehabilitation. J Electromyogr Kinesiol. 2011; 21(2):220-8. [DOI:10.1016/j.jelekin.2011.01.002] [PMID]

[32] Dolak KL, Silkman C, McKeon JM, Hosey RG, Lattermann C, Uhl TL. Hip strengthening prior to functional exercises reduces pain sooner than quadriceps strengthening in females with Patellofemoral Pain Syndrome: A randomized clinical trial. J Orthop Sport Phys Ther. 2011; 41(8):560-70. [DOI:10.2519/ jospt.2011.3499] [PMID]

[33] Korman P, Straburzyńska-Lupa A, Rutkowski R, Gruszczyński J, Lewandowski J, Straburzyński-Lupa M, et al. Kinesio taping does not alter quadriceps isokinetic strength and power in healthy nonathletic men: A prospective crossover study. Biomed Res Int . 2015; 2015(626257):1-5. [DOI:10.1155/2015/626257] [PMID] [PMCID]

[34] Van der Heijden RA, Lankhorst NE, van Linschoten R, BiermaZeinstra SM, van Middelkoop M. Exercise for treating patellofemoral pain syndrome. Cochrane Database Syst Rev. 2015; 1:CD010387. [DOI:10.1002/14651858.CD010387.pub2]

[35] Collins NJ, Barton CJ, van Middelkoop M, Callaghan MJ, Rathleff MS, Vicenzino BT, et al. 2018 Consensus statement on exercise therapy and physical interventions (orthoses, taping and manual therapy) to treat Patellofemoral pain: Recommendations from the $5^{\text {th }}$ International Patellofemoral Pain Research Retreat, Gold Coast, Australia, 2017. Br J Sports Med. 2018; 52(18):1170-8. [DOI:10.1136/bjsports-2018-099397] [PMID]

[36] Freedman SR, Brody LT, Rosenthal M, Wise JC. Short-term effects of patellar kinesio taping on pain and hop function in patients with Patellofemoral Pain Syndrome. Sports Health. 2014; 6(4):294-300. [DOI:10.1177/1941738114537793] [PMID] [PMCID]

[37] Aghapour E, Kamali F, Sinaei E. Effects of Kinesio Taping $(R)$ on knee function and pain in athletes with Patellofemo- ral Pain Syndrome. J Bodyw Mov Ther. 2017; 21(4):835-9. [DOI:10.1016/j.jbmt.2017.01.012] [PMID]

[38] Logan CA, Bhashyam AR, Tisosky AJ, Haber DB, Jorgensen $A$, Roy $A$, et al. Systematic review of the effect of taping techniques on Patellofemoral Pain Syndrome. Sports Health. 2017; 9(5):456-61. [DOI:10.1177/1941738117710938] [PMID] [PMCID]

[39] Keet JHL, Gray J, Harley Y, Lambert MI. The effect of medial patellar taping on pain, strength and neuromuscular recruitment in subjects with and without patellofemoral pain. Physiotherapy. 2007; 93(1):45-52. [DOI:10.1016/j.physio.2006.06.006] 